\title{
Plus de chiens dans le secteur de la santé!
}

\section{Rouven Porz}

Privat-docent, docteur ès lettres, biologiste dipl., responsable de la cellule Ethique clinique de I'Insel Gruppe AG (Berne), chercheur à I'Université populaire d'Amsterdam, secrétaire général de I'European Association of Centres of Medical Ethics (EACME) et membre de la rédaction éthique du BMS

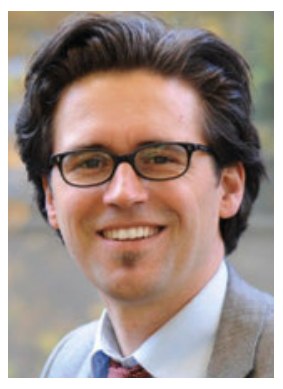

Je trouve que nous avons besoin de plus de chiens dans le secteur de la santé. Je ne parle pas de chiens de thérapie ou à caresser (même si ce ne serait sans doute pas un mal non plus), mais de ma tentative de construire ici une métaphore. Pour celle-ci, je classe les gens en deux catégories, les "chiens» et les «singes». Je veux dire que certaines personnes ressemblent davantage à des chiens, d'autres à des singes. Ou, pour donner le fond de ma pensée: nous avons besoin de plus de chiens! Et d'un peu moins de singes, merci. Voilà à quoi je veux en venir. Pardonnez-moi si la métaphore est parfois un peu bancale, c'est le propre de la plupart d'entre elles. Je vais néanmoins tenter de la filer autant que possible. C'est parti! Ouaf, ouaf! Les chiens ne savent pas mentir. Ils ne sont pas capables de simuler. Ils sont toujours dans le présent, dans l'ici et maintenant de leurs sentiments et intuitions. Ils suivent la meute, leur comportement est fiable et prévisible. Certains sont même des chefs de meute, des individus responsables. D'un point de vue éthique, ces caractéristiques canines pourraient être résumées par des valeurs: authenticité, loyauté et sincérité.

\section{Les chiens ne savent pas mentir.}

Mais voilà, l'homme ne descend pas du chien, mais du singe. Les hominidés, singes anthropoïdes. Ils ont évolué dans une autre direction. Conscience, capacité de réflexion, outils, auxquels il faut ajouter un pouce opposable et un cerveau dont le grossissement évoque vaguement celui d'un furoncle. Ces développements fantastiques en soi nous ont permis d'évoluer culturellement. Mais ce sont aussi eux qui nous ouvrent les portes du raisonnement stratégique, donc de la duperie et du mensonge.

Les chiens ont une capacité de réflexion moindre et sont dépourvus de conscience d'eux-mêmes lorsqu'ils se regardent dans la glace. Ils n'ont pas non plus de buts cachés, de desseins inavoués (hidden agendas). Ils ne peuvent pas faire semblant.

Dans le cadre de mon travail d'éthicien clinique, je propose des discussions de cas. Elles doivent aider les par- ticipants à reconsidérer les valeurs liées à leur propre rôle professionnel et à mettre au point les meilleures solutions à des situations cliniques difficiles, telles des interruptions de traitement ou de grossesse, des décisions en fin de vie, etc. Une étape importante de ces discussions de cas est le "changement de perspective», une tentative méthodiquement dirigée d'adopter le

\section{Mais voilà, l'homme ne descend pas du chien,} mais du singe.

point de vue de la personne dont on n'arrive tout simplement pas à comprendre les souhaits et valeurs. Il s'agit de se mettre à la place de l'autre. Les singes sont doués pour cela, les chiens en sont incapables. Ces derniers ne peuvent jamais se départir de leur propre perspective. Le fait d'assumer ses propres valeurs est une autre étape importante de ces discussions de cas. Qu'est-ce qui t'importe dans ton métier? Qu'est-ce que tu défends? Dans le cas précis de la décision que nous devons prendre tous ensemble ici. Les chiens sont capables de répondre, les singes n'osent généralement pas. Et c'est là le fond du problème.

Les chiens assument toujours courageusement leur opinion. Les singes se demandent pour la plupart si elle s'inscrit dans la stratégie globale et s'il ne serait pas préférable de prétendre avoir un avis différent. Bref, l'authenticité se perd - c'est du moins mon impression. Mais je me trompe peut-être. Après tout, je ne suis qu'un chien.

C'est sûr, les accomplissements des chiens en termes de civilisation sont plus modestes. Nous n'avons même pas construit d'hôpitaux universitaires. Nous ne sommes pas non plus allés de manière autonome sur la lune. C'est là que ma métaphore coince un peu. N'empêche qu'en termes de loyauté et d'authenticité, les singes auraient encore beaucoup à apprendre de nous.

Remarque: Cette métaphore a déjà été présentée dans le numéro $1 / 13$ de Punkt, la revue du personnel de l'Inselspital, page 25. 